\title{
Tariq Rahman, From Hindi to Urdu. A Social and Political History
}

Karachi, Oxford University Press, 2011, XX, 456 p.

Denis Matringe

\section{(Q) OpenEdition}

\section{Journals}

Édition électronique

URL : http://journals.openedition.org/assr/24702

DOI : $10.4000 /$ assr.24702

ISSN : $1777-5825$

Éditeur

Éditions de l'EHESS

Édition imprimée

Date de publication : 30 décembre 2012

Pagination : 265

ISSN : 0335-5985

Référence électronique

Denis Matringe, "Tariq Rahman, From Hindi to Urdu. A Social and Political History », Archives de sciences sociales des religions [En ligne], 160 | octobre-décembre 2012, mis en ligne le 27 mars 2013, consulté le 10 décembre 2020. URL : http://journals.openedition.org/assr/24702 ; DOI : https:// doi.org/10.4000/assr.24702

Ce document a été généré automatiquement le 10 décembre 2020.

(c) Archives de sciences sociales des religions 


\section{Tariq Rahman, From Hindi to Urdu. A Social and Political History}

Karachi, Oxford University Press, 2011, XX, 456 p.

Denis Matringe

\section{RÉFÉRENCE}

Tariq Rahman, From Hindi to Urdu. A Social and Political History, Karachi, Oxford University Press, 2011, XX, 456 p. 
1 La République islamique du Pakistan, née, comme la République indienne, en 1947 de la terriblement sanglante partition de l'Inde britannique (douze millions et demi de personnes déplacées, plusieurs centaines de milliers de morts, peut-être un million), présente un certain nombre de caractéristiques singulières. La plus connue tient à sa naissance même, dans la foulée d'un mouvement séparatiste qui vit un parti politique, la Ligue musulmane, et ses partisans obtenir la création d'un État censé être celui des musulmans de l'ancienne Inde britannique, quand bien même la majorité des musulmans ne vivant pas dans les régions appelées à faire partie du Pakistan choisirent de rester dans la République indienne. Une autre singularité du Pakistan provient de ce qu'il adopta comme langue officielle

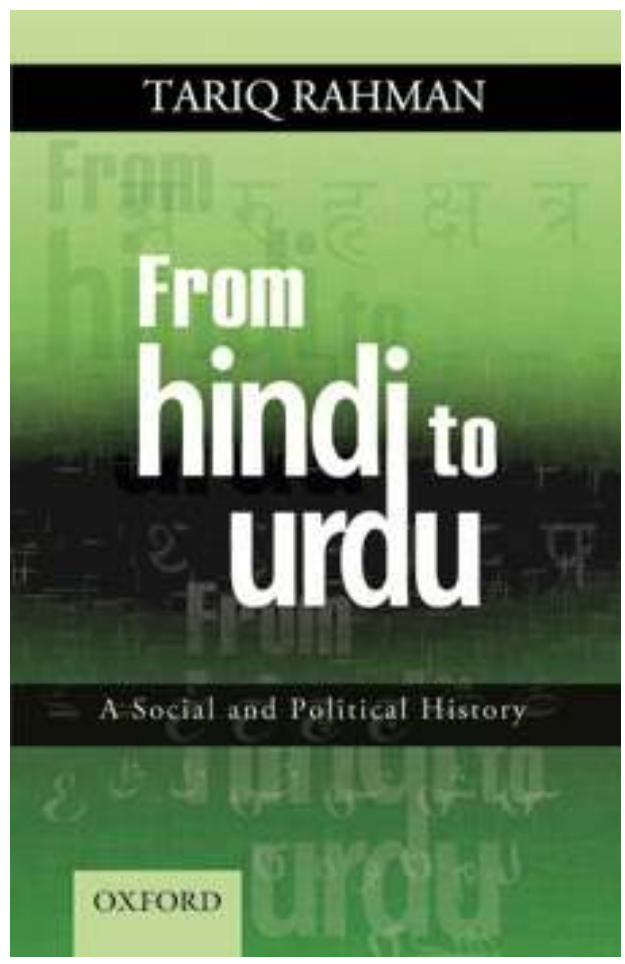
l'ourdou, devenu la principale langue de culture des musulmans de l'Inde au début du XVIII siècle et l'une de leurs langues religieuses, se substituant graduellement dans ce rôle au persan - mais langue maternelle des seuls musulmans cultivés de la grande aire linguistique hindi qui s'étend autour de Delhi des frontières du Panjab, du Sind et du Gujarat à celle du Bengale, et du piémont himalayen aux monts Vindhya et à la Narmada. Au Pakistan, l'ourdou est donc la langue maternelle des seuls réfugiés en provenance de ces régions, et à vrai dire, principalement de l'actuel Uttar Pradesh - le Bihar, le Rajasthan et le Madhya Pradesh n'ayant pratiquement pas été concernés par l'émigration musulmane au moment de la partition. En conséquence, l'ourdou est aujourd'hui déclaré comme langue maternelle par plus de quarante-huit millions d'Indiens et par seulement onze des près de deux cents millions de Pakistanais.

Grammaticalement et par leur vocabulaire fondamental, le hindi et l'ourdou sont une seule et même langue, lointainement issue du sanskrit comme les autres langues indoaryennes (panjabi, bengali, marathi, etc.) et comme les langues romanes le sont du latin : les livres de référence sur cette évolution sont la magistrale synthèse d'un ancien professeur au Collège de France, Jules Bloch (1880-1953) ${ }^{1}$ et le grand dictionnaire étymologique de Ralph Turner (1888-1983), professeur de sanskrit à la School of Oriental and African Studies de Londres ${ }^{2}$. Mais pour des raisons culturelles, le hindi s'écrit dans la devanagari du sanskrit, langue à laquelle il fait tous ses emprunts savants, tandis que l'ourdou est noté en alphabet arabe et recourt à l'arabe et au persan pour ses lexiques spécialisés. Les affrontements intercommunautaires qui ont marqué le mouvement pour l'indépendance dans l'Inde britannique se sont accompagnés de vives controverses linguistiques entre partisans du hindi et thuriféraires de l'ourdou, et le conflit continue par-dessus la frontière indo-pakistanaise ainsi qu'en Inde même, où la place de l'ourdou est en forte régression, bien que cette langue ait été reconnue par la constitution indienne, promulguée en 1950, comme l'une des langues nationales de la 
fédération. Cette situation est résumée de manière saisissante par Robert D. King (Nehru and the Language Politics of India, Delhi, Oxford University Press, 1998, p. 84) :

Communal hatreds between Muslims and Hindus cannot be simply wished away by pretending that the scripts used to write their language are devoid of evoked meaning. The power of language as icon must never be underestimated. Like it or not, the Urdu script means Muslim, the Devanagari script means Hindu. The Urdu script as seen by an angry Hindu mob summons up talismanic images from the present and the past: cowslaughter, temple-bashing, iconoclasm, crescent and star, Aurangzeb, green. When Hindus bent on doing violence to Muslims see a shop sign in Urdu, they want to smash it and burn that shop down. And vice-versa. The Hindi script conveys to an enraged Muslim mob Vishnu and Shiva and a score of many-handed, many-headed gods and goddesses, cowdung, music before the mosque, dead pigs flung into mosques, Shivaji. Old sins cast long shadows. Nowhere is this truer than in India's burning sun.

Les rapports entre ces deux langues sœurs devenues ennemies intimes, l'histoire de leur naissance et de leur développement et les affrontements dont elles ont été l'enjeu ont fait l'objet d'une abondante littérature savante, dont le champ, jusqu'à l'ouvrage de Tariq Rahman, a été balisé ces quarante dernières années, par cinq livres importants. Le premier est le travail pionnier de Kerrin Dittmer, Die indischen Muslims und die HindiUrdu-Kontroverse in den United Provinces (Wiesbaden, Otto Harrossowitz, 1972). Après y avoir présenté les United Provinces (Awadh et Agra) de l'Inde britannique ainsi que leur population musulmane et ses pratiques littéraires et linguistiques, l'auteur étudie en grand détail la naissance de la controverse entre hindi et urdu, en prêtant beaucoup d'attention, à propos de l'urdu, d'une part à la dimension institutionnelle du conflit, avec de riches développements sur les centres d'instruction tant britanniques qu'indiens, et d'autre part au rôle des associations. La dernière partie de son livre est consacrée à la question, âprement débattue pendant le mouvement national indien, de savoir quelle serait la langue nationale de l'Inde: le hindi, l'ourdou ou leur base commune appelée hindustani.

5 La question de l'ourdou a été reprise dans une étude devenue le classique de référence sur la question: Paul Brass, Language, Religion and Politics in North India (Cambridge, Cambridge University Press, 1974). L'apport de ce livre est triple. D'une part, Brass, dans la deuxième partie (p. 51-274), après avoir résumé les polémiques antérieures à l'indépendance, y suit le destin de l'ourdou en Inde de 1947 au début des années 1970 (p. 182-274), concentrant son attention sur le lien entre les revendications identitaires des musulmans indiens, leurs formes d'organisation politique et l'ourdou. D'autre part, son analyse est encadrée dans l'ouvrage par l'étude d'un mouvement linguistique au Bihar en faveur du maithili (un «dialecte» du hindi) et par celle du mouvement au terme duquel les sikhs du Panjab obtinrent en 1966, en abritant leurs revendications socioreligieuses derrière une exigence linguistique, la division du Panjab indien en trois états : Panjab, Haryana et Himachal Pradesh. Le hindi restait la langue nationale dans le Haryana et le Himachal Pradesh, tandis le panjabi devenait la langue nationale du nouveau Panjab dans lequel, à la faveur de cette partition, les sikhs se retrouvaient majoritaires (60\% de la population). Enfin, Brass donne à ses trois essais un cadre conceptuel unique : la théorie de Karl Deutsch (Nationalism and Social Communication: an Enquiry into the Foundation of Nationality, Cambridge, Mass., MIT Press, 1966) selon laquelle l'assimilation progresse quand la mobilisation des groupes minoritaires est contenue, tandis que c'est la différentiation qui l'emporte en cas contraire. C'est cette 
mobilisation qui intéresse Brass au premier chef, et notamment la manière dont des groupes minoritaires en lutte autour d'enjeux liés à l'ethnicité organisent leur combat, dans le contexte de l'Inde nehruvienne, autour d'un symbole - en l'occurrence linguistique - acceptable par l'État fédéral.

Le livre de Brass fut une source d'inspiration pour un travail tout aussi remarquable consacré vingt ans plus tard au hindi par Christopher R. King, One Language, Two Scripts: The Hindi Movement in North India (Bombay, Oxford University Press, 1994). Travaillant dans le même cadre deutschien que Brass, King ne cherche pas à faire œuvre de théoricien, mais s'attache, sur la base d'une énorme documentation (rapports officiels, textes en hindi, littérature secondaire) à retracer l'histoire de la création et de la propagation du hindi comme rival de l'ourdou au cours du xIXe siècle. Il met clairement en équation cette évolution en la présentant, dans le chapitre I, comme un processus " in which supporters of Hindi strove to transform the existing equations of Urdu $=$ Muslim + Hindu and Hindi $=$ Hindu + Muslim into Urdu = Muslim and Hindi $=$ Hindu $»($ p. 15). Les trois chapitres suivants illustrent chacun un aspect particulier de cette histoire, tout en en suivant la chronologie. La naissance du hindi résulte de la politique britannique d'enseignement des langues dans le Fort William College fondé à Calcutta en 1800, où des manuels séparés sont produits en ourdou et dans cette langue, qui s'impose comme médium autonome d'abord pour la prose puis, contre le braj (variante occidentale et langue de la poésie krishnaïte), pour la poésie. Signalons ici, sans entrer dans les détails, que de grands textes de l'hindouisme ont été écrits en hindi à partir du $\mathrm{xv}^{\mathrm{e}}$ siècle.

7 Le chapitre III est consacré aux méandres de la politique linguistique des Britanniques après qu'ils ont remplacé, en 1837, le persan comme langue officielle d'abord par l'ourdou. Le chapitre IV, consacré aux relations entre langue, éducation et emploi dans le dernier $\mathrm{xIX}^{\mathrm{e}}$ siècle, expose les contradictions entre une politique officielle semblant favoriser l'ourdou et un système scolaire produisant un nombre croissant d'Indiens éduqués en hindi et qui cherchent à promouvoir cette langue quelle que soit leur caste (à l'exception des hindous de la caste des kayasths, gagnant traditionnellement leur vie dans l'administration du fait de leur connaissance du persan et de l'ourdou). Le cinquième chapitre est consacré au mouvement pour le hindi, aux organisations qui en furent le fer de lance à la fin du XIX siècle et aux campagnes pour l'adoption du hindi comme langue officielle au niveau provincial dans la région qui forme aujourd'hui l'Uttar Pradesh. King est très conscient qu'il est difficile, quand on traite d'un tel sujet, d'éviter tout parti-pris, et son propre rejet des extrémistes contemporains de l'ourdou et du hindi apparaît tant dans le "one language» du titre de son livre que dans le plaidoyer contre l'absolutisme des partisans de la "pureté » (śuddhatā) du hindi dans l'Inde contemporaine et dans son appel à ne pas oublier que nombre d'hindous restent de fervents aficionados de l'ourdou.

Mais la volonté de réunir ce qui a été séparé peut faire d'un ouvrage à prétention savante un livre militant. Tel avait été le cas, dix ans après le livre de Brass, de l'étude ambitieuse publiée par un écrivain hindi renommé, Amrit Rai, sous le titre A House Divided: The Origin and Development of Hindi/Hindavi (Delhi, Oxford University Press, 1984). Ambitieux parce que Rai entend y retracer toute l'histoire du hindi et de l'ourdou de l'époque du Veda (les premières compositions sanskrites remontant à la première moitié du deuxième millénaire avant l'ère chrétienne) jusqu'au $\mathrm{xx}^{\mathrm{e}}$ siècle, l'accent étant mis, toutefois, sur la période allant du $\mathrm{xl}^{\mathrm{e}}$ siècle, quand apparaissent selon 
l'auteur les premières attestations du vieil hindi commun ou hindavi, au XVIII ${ }^{\mathrm{e}}$ siècle où, d'après Rai, se produit la fracture avec l'apparition d'un ourdou hautement persanisé. Mais ambigu, parce que l'étude, truffée de longues citations non traduites (près d'un tiers du livre!), tourne en réalité autour d'un thème central marqué par la nostalgie d'une mythique unité perdue entre hindous et musulmans. Rai considère comme artificielle et désastreuse sur le plan socioculturel la bifurcation linguistique intervenue avec l'évolution séparée de l'ourdou quand six siècles durant le hindavi avait été la langue de culture commune des hindous et des musulmans de l'Inde du Nord. Il est convaincu de même que la production, au XIX et au $\mathrm{Xx}^{\mathrm{e}}$ siècle, d'un hindi délibérément très sanskritisé est une grave erreur.

9 La même longue histoire est reprise de manière concise et lumineuse sur un ton académique sans parti-pris, mais non dépourvu d'humour et de sensibilité, par deux des meilleurs spécialistes occidentaux respectivement de l'ourdou et du hindi, à l'époque tous deux enseignants à la School of African and Oriental Studies (SOAS) de Londres, Christopher Shackle et Ruppert Snell. Le fruit de leur collaboration est un merveilleux manuel intitulé Hindi and Urdu since 1800: A Common Reader (Londres, SOAS, 1990) comportant introduction, grammaire, anthologie et lexique, dans lequel l'introduction remonte, de manière savante et rigoureuse, jusqu'à l'origine sanskrite de l'ourdou et du hindi, et dont les textes retenus portent précisément sur l'univers spécifique de l'ourdou et du hindi et sur le rôle respectif de ces langues dans la société de l'Inde du Nord et aussi, après 1947, du Pakistan.

10 Tels sont les hauts lieux qui, au cours de ces quarante dernières années, ont balisé le paysage dans lequel s'inscrit le livre de Tariq Rahman, écrivain et «Distinguished National Professor» à l'Université Qaid-e-Azam d'Islamabad au Pakistan. Le champ couvert par son livre a une amplitude qui rappelle celui d'Amrit Rai, comme aussi le rêve de l'unité retrouvée entre hindi et ourdou, sur lequel nous reviendrons à propos de la conclusion. Mais à la différence de celui de Rai, l'ouvrage de Rahman est centré sur l'origine et le développement de l'ourdou, avec une perspective beaucoup plus vaste que celle de Dittmer et Brass.

11 La question de départ est de savoir quand et pourquoi une langue qui, du XIII ${ }^{e}$ au XVIII siècle était principalement appelée hindi (hindvi, hindavi), est devenue l'ourdou. Dans les chapitres II, «Names» (p. 18-54), III «Age» (p. 55-78) et IV "Origins and historiography» (p.79-97), Rahman, partant du constat que l'origine de la langue aujourd'hui appelée ourdou est le hindi, mais un hindi ancien (hindvi) bien différent de celui de l'Inde contemporaine qui en est de même issu, affirme que cette évolution tient à des causes à la fois « naturelles » et « humaines ».

12 Concernant l'évolution naturelle, la langue appelée hindvi par les premiers conquérants musulmans de l'Inde était, selon Rahman, parlée dans la plus grande partie de l'Inde du Nord et elle est celle que parlait, par exemple, le grand poète mystique indo-musulman de Delhi, Amir Khusrau (1253-1324), et dans laquelle il écrivit certains de ses vers (l'essentiel de son œuvre étant en persan). La langue quotidienne et commune des Indiens du nord appelée hindoustani à l'époque coloniale serait le descendant « naturel » de ce hindvi. Rahman réfute donc l'idée commune selon laquelle l'ourdou (dont le nom signifie « camp [militaire] » en turc) serait apparu comme lingua franca des soldats de diverses origines qui se côtoyaient dans les armées du sultanat de Delhi puis de l'Empire moghol. Si tel était le cas, l'ourdou serait un pidgin ou un créole. Or, il se présente comme une langue très sophistiquée sur le plan linguistique, résultat 
d'un contact culturel de cinq cents ans ( $\mathrm{XIII}^{\mathrm{e}}-\mathrm{XVIII}{ }^{\mathrm{e}}$ siècles) entre hindous et musulmans, les premiers l'écrivant dans la devanagari du sanskrit et les seconds en alphabet arabe. Rahman réfute aussi l'hypothèse, formulée par le savant panjabi Hafiz Mahmud Shirani dans un livre en ourdou resté fameux, Panjāb mem urdū (Lahore, 1928), « l'ourdou dans le Panjab»(p.87-89), selon laquelle l'ourdou se serait d'abord développé dans le Panjab.

13 Fort de ces constats, l'auteur avance une nouvelle hypothèse pour expliquer l'appellation d'«ourdou» finalement donnée à une certaine variante du hindvi. Son nom complet, zabān-i urdū-yi mu'allā, signifierait non « la langue du camp très haut ", comme il est communément admis, mais « la langue de la cité très haute », c'est-à-dire de Delhi, et cette expression, comme sa forme abrégée "urdú», désignerait plus précisément l'idiome artificiel, riche de nombreux emprunts au persan et, à travers lui, à l'arabe, développé sur la base du hindvi par les élites musulmanes de cette ville à partir du XviII ${ }^{e}$ siècle. Pour Rahman, l'ourdou est donc le produit d'un processus de standardisation qui l'a définitivement associé à l'identité musulmane en Inde du Nord (chap. V, «The Islamization of Urdu », p. 98-135). Il s'agit là d'une thèse centrale du livre. Notons au passage le destin extraordinaire du substrat commun au hindi et à l'ourdou, tous deux lointainement issus du sanskrit : tandis que le hindi se situe dans la continuité de ses origines, tant sur le plan linguistique que religieux, l'ourdou, lui, est devenu par excellence l'idiome indien porteur d'une culture religieuse dont la langue de référence est l'arabe.

On pourra hésiter à suivre l'auteur dans son interprétation du nom urdū, apparenté au français « horde ", qui signifie clairement « camp » et non " cité ", sauf peut-être par métaphore pour le camp-ville de l'empereur moghol en campagne militaire, mais on retiendra de son argumentation la notion d'une disjonction entre une sorte de koinè des camps et des bazars qui reste vivace à grande échelle aujourd'hui au Panjab, au Sind et dans toute la ceinture hindi de l'Inde et l'ourdou, langue de haute culture islamique qui s'est développée à partir du XviII ${ }^{e}$ siècle.

Concernant la suite de l'ouvrage, une distinction peut être établie entre trois types de chapitres. Les chapitres VIII, «The British and Hindustani » (p. 199-226), IX « Urdu in the Princely States» (p. 227-260) et X «Urdu as the Language of Employment» (p. 261-278) reprennent à nouveaux frais l'histoire bien connue et en partie racontée dans les livres de Dittmer et King sur le rôle joué par les Britanniques dans l'évolution et la diffusion de l'ourdou à travers leur politique linguistique, leurs institutions et leurs classifications rigides particulièrement sensibles dans l'administration de la justice d'une part et les grilles de leurs recensements décennaux d'autre part. Notons ici que le chapitre IX met judicieusement en perspective la situation du Cachemire et de Hyderabad où, du fait d'une population à majorité musulmane, l'ourdou remplaça le persan comme langue officielle et devint un symbole identitaire fort pour les musulmans locaux comme pour ceux venus de l'extérieur. Le contraste est frappant avec les états princiers majoritairement hindous, où s'imposèrent diverses variantes de hindi.

16 Les chapitres VI «Urdu as an Islamic language » (p. 136-163) et VII « Urdu as the Language of Love » (p. 164-198) insistent de manière originale sur les deux grands domaines de la littérature ourdoue : la religion et l'amour. Le chapitre VII, après avoir longuement analysé les divers aspects, les styles et les genres - au premier rang desquels le ghazal - 
de la thématique amoureuse et érotique dans la littérature ourdoue, se conclut sur le constat attristé de son recul au Pakistan :

[...] the amorous and erotic associations were suppressed in the wake of the political urgency and identity politics of the Pakistan movement, and then the perpetual struggle the Pakistani ruling elite waged against the ethnic elites of Pakistan on the one hand, and the Western and Indian "Other" on the other (p.197).

L'histoire du renforcement de l'association entre ourdou, islam et politique fait l'objet du chapitre VI. L'auteur y relate la conquête des territoires du religieux par l'ourdou, des mouvements de réforme du XIX ${ }^{\mathrm{e}}$ siècle à l'enseignement dans les madrasas et au soufisme. Mais après 1947, l'évolution est différente en Inde, où l'ourdou est en déclin face au hindi même chez les musulmans, et au Pakistan, où il est langue officielle et au cœur d'une idéologie nationale parfois résumée en un slogan : un peuple, une religion, une langue. Rahman remarque en outre: "While in Pakistan, Urdu is often associated with pro-establishment and right-wing forces, in India, it is anti-establishment and generally stands for the autonomy, identity and rights of the Muslim community" (p.159). Le chapitre se termine avec des pages fortes sur les liens, au Pakistan, entre élite panjabi, ourdou, identité musulmane et politique linguistique, et la situation est ainsi résumée, non sans humour :

In short, Urdu and Islam are used to subordinate the ethnic elites in favour of the Punjabi elite in Pakistan but, ironically enough, both are in fact subordinated to the interests of the Westernized, English-using urban elite (p. 162).

Rappelons que la promotion de l'ourdou comme langue de la nation et de l'islam au Pakistan, au détriment des langues régionales (panjabi, sindhi, pashto, baloutche, cachemiri, etc.) et des cultures populaires dont elles sont porteuses, a fait l'objet d'une étude détaillée par Tariq Rahman: Language and Politics in Pakistan (Karachi, Oxford University Press, 1996).

21 Avec ce déclin de l'ourdou comme véhicule de la poésie amoureuse et le recentrement de cette langue sur l'expression d'un religieux tenant souvent de la vulgate islamique que les wahhabites saoudiens cherchent à imposer partout dans le monde musulman à grand renfort de pétrodollars, le Pakistan est bien dans ce qu'Olivier Roy appelle «le temps de la religion sans la culture » (La sainte ignorance : la religion sans la culture, Paris, Le Seuil, 2008).

22 Enfin, les chapitres XI à XIV donnent au livre de Rahman toute son originalité et couronnent parfaitement l'ambition annoncée dans l'introduction de faire une histoire non seulement politique, mais aussi et surtout sociale de l'ourdou (p.5). Sur la base d'une énorme documentation indienne et pakistanaise, notamment des données statistiques, ils retracent l'utilisation de l'ourdou, dans l'Inde coloniale puis dans l'Union indienne et au Pakistan, dans les domaines de l'éducation (p. 278-316), de la presse et de l'édition (p.317-347), de la radio (p. 348-366) et enfin dans ceux du cinéma et de la télévision (p. 366-389).

Dans la conclusion de son livre (p.389-399), Rahman, après être revenu sur sa démarche d'ensemble, s'interroge sur l'avenir de l'ourdou, et aussi sur celui du hindi, avec d'intéressantes considérations sur les usages de ces langues et leur évolution spécifique en contexte diasporique. Les efforts, au demeurant très limités, des gouvernements indiens et pakistanais pour promouvoir l'apprentissage des variantes formelles des deux langues sont de peu de poids face à l'utilisation par les locuteurs 
indiens et pakistanais de leur base commune hindoustani pour communiquer, notamment entre eux, par-delà leur frontière transplantée.

Rahman termine sa belle et riche étude par l'expression du rêve de l'unité retrouvée entre hindi et ourdou. Ce rêve en dit un autre : celui de la coexistence harmonieuse entre hindous et musulmans dans une Asie du Sud où il serait possible de réunir ce que la Partition de 1947 a séparé, si seulement pouvaient disparaître les obstacles à la paix que sont la querelle à propos du Cachemire, le terrorisme et la question du partage des eaux (p. 398). Cet idéal peut sembler bien hors de portée, mais selon Rahman, les intellectuels locuteurs de l'ourdou ont un rôle à jouer pour œuvrer à son avènement : What is in the hands of the scholars is to debunk the myths which link Urdu with military origins or deny that words of Hindi - such common words as prem [amour], sāgar [océan], nainā [œeil], chintā [pensée] - are part of the heritage of Urdu. It is, after all, only the truth to say that even now, after about two hundred years of separation and drifting apart - spoken Urdu and Hindi are the same language. It is only by not losing sight of the continuities and shared cultural features among Pakistanis and (north) Indians that we can hope to transcend the mutual hatred which threatens to annihilate this ancient land (p. 399).

\section{NOTES}

1. Indo-Aryan from the Vedas to Modern Times (trad. du français par Alfred Master, éd. revue et corrigée, Paris, Adrien Maisonneuve, 1965).

2. A Comparative Dictionary of the Indo-Aryan Languages (4 vols., vols. 1-3, Oxford, Oxford University Press, 1966-1971; vol. 4, Londres, School of Oriental and African Studies, 1985). 